\title{
Fine Structure and Histochemistry of the Teeth of the Tree Frog (Hyla japonica)
}

\author{
By \\ Iwao SATO $^{{ }_{1}}$, Kazuyuki SHIMADA ${ }^{{ }_{2}}$, Tooru SATO $^{{ }_{1}}$ and Tadashi KITAGAWA ${ }^{{ }_{2}}$ \\ ${ }^{1)}$ Department of Anatomy, School of Dentistry at Tokyo. The Nippon Dental University, Tokyo, Japan*1 \\ ${ }^{2)}$ Department of Anatomy, Nihon University School of Dentistry, Tokyo, Japan ${ }^{2}$ \\ - Received for Publication, August 25, 1989-
}

Key Words: Amphibian, Tooth, Enamel, Histochemistry

\begin{abstract}
Summary: Observations were carried out on the morphology of the teeth of the adult tree frog (Hyla japonica) at an ultrastructural level. Many small crystals pile up in the enamel layer which do not typical enamel rods. At the thin layered buccolingual region of the tooth, the matrices of crystals are arranged either regularly in the enamel layer or randomly in the dentine layer. In particular, the apical portion of the enamel layer is more regularly arranged and mineralized. ATPase activity is located in multivesicular bodies, the round mitochondria and the lateral borders forming the infolding of the interdigitation of the ameloblast. The reaction is also found in small round vesicles in the enamel layer and the distal cell membrane during the maturation stage. During enamel formation of teeth of Hyla japonica, there is no Tomes' process. Instead secretory granular bodies contain ATPase, may control calcium entry into developing enamel of the ameloblasts. The content of ATPase is less than that of mammals. In contrast, the Tomes' process, containing much ATPase, is related to the pattern and arrangement of the enamel matrices in mammals.
\end{abstract}

Enamel matrices of amphibians do not form typical enamel rods which is resemble to a prismless zone is found during the developmental stage of enamel layer in rats (Ishida et al., 1983). In amphibians there are no Tomes' processes during tooth development. In adult urodeles, cells of the inner dental epithelium do not produce them during the secretion of the enamel layer (Meredith Smith and Miles, 1971). It might be speculated that the lack of typical enamel rods is the result of the failure of inner epithelium cells to produce Tomes' processes during tooth development (Casey and Lawson, 1981), for the Tomes' process is concerned with the pattern and arrangement of enamel matrices.

On the other hand matured ameloblasts might control calcium $(\mathrm{Ca})$ entry into the developing enamel, a process inferred by the Ca-ATPase distributed over the plasma membrane of secretory ameloblasts, including the Tomes' processes, in the rat incisor. When Tomes' processes are lost, the Ca-ATPase reaction also disappears from the distal plasma membrane of the ameloblasts (Crenshaw and Takano, 1982). Thus we pose the questions: how do amphibians without Tomes' processes control Ca distribution and where is ATPase found?

The present work describes the ultrastructure and arrangement of matrices of the enamel layer, and the distribution of Ca-ATPase during formation of enamel, in teeth of adult Hyla japonica.

\section{Material and methods}

Six adults (body weight $1.2-2.0 \mathrm{~g}$ ) of Hyla japonica were used. Teeth were removed from the upper jaw and prefixed in $0.2 \mathrm{M}$ glutal-cacodylate $(\mathrm{pH} 7.2)$ and postfixed with $1 \%$ osmic acid for $1 \mathrm{hr}$. at $4{ }^{\circ} \mathrm{C}$. The material was dehydrated in ethyl alcohol, and finally embedded in epoxy resin (Epon-812). Silver-gray sections were stained with uranyl acetate and then with lead citrate. Sections were observed under a Hitachi H-700 and JEM 100-B electron microscope (TEM). Other teeth were removed and fixed for 1 week at $4^{\circ} \mathrm{C}$ in $0.2 \mathrm{M}$ glutalcacodylate $(\mathrm{pH} 7.2)$, dehydrated in ethyl alcohol and dried by a critical point drying method (methylacetate and carbonic acid gas). A $1.5 \mathrm{~nm}$ thick layer of Au-Pd was vacuum-evaporated on the surface of the dried specimens. Coated specimens were observed under a Hitachi X-560 scanning electron microscope (SEM). Fresh teeth germ sections $(50-100 \mu \mathrm{m})$ cut at $40 \mu \mathrm{m}$ thickness, were fixed in $2 \%$ paraformaldehyde in $0.1 \mathrm{M}$

Correspondence on this article should be addressed to Dr. Kazuyuki Shimada, Department of Anatomy, Nihon University School of Dentistry, 1-8-13 Kanda-Surugadai, Chiyoda-ku. Tokyo 101 JAPAN

Telephone 03-293-5711 Ext. 226 


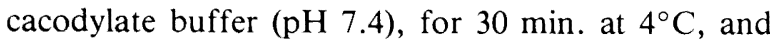
washed in the same buffer for $30 \mathrm{~min}$. ATPase was demonstrated using the Ando' method (Ando et al., 1981). After incubation, teeth germ material was dehydrated in ethyl alcohol and finally embedded in epoxy resin (Epon-812). Silver-gray sections were stained with uranyl acetate and observed under a Hitachi H-700 and a JEM 100-B electron microscope (TEM).

\section{Results}

(A) Scanning electron microscopy of the teeth (Fig. 1)

The teeth of the upper jaw and palate are uniformly bicuspid in form, each tooth curved slightly backwards with an elongated apex. The apex of the tooth has two cusps, a lingual cusp cone-shaped and elongated and a labial one flattened (Fig. 2). They have a smooth surface up to the edge of the pedicel-enamel junction, except for the lingual surface of the lingual cusp which has a rough surface (Fig. 3 ). In the enamel layer, there are piles of fine stake-like structures leading on to the dentine layer which are scattered in many oval masses (Fig. 4). In the dentine layer and the basal region near the pedicel, many small oval masses are piled up (Figs. $5,6)$. The junction of the tooth and pedicel consists of collagen fibers (Fig. 7).

(B) Transmission electron microscopy of the teeth (Fig. 1)

The enamel varies in thickness, about 3-5 $\mu \mathrm{m}$ thick at the cusp, gradually reducing in thickness to disappear near the basal region of the tooth (Fig. 1). The fine structure of the enamel of the cusp differs from that at the basal region near the pedicel. In the cusp layer, mineral crystals orientate at right angles to the long axis of the tooth (Fig. 8). Furthermore, in this layer, the mineralized fibrous matrices extend randomly to the dentine-enamel junction and the enamel layer. In the basal layer near the pedicel, the mineral crystal matrices orientate at right angles to the long axis of the tooth, those of the enamel layer near the tooth showing a variety of angles (Fig. 9). Near the tooth surface, crystals form small fine spindles and at the inner layer form small masses. In the pedicel-enamel junction, the enamel layer is less mineralized than in other layers of the tooth; hence the crystal density is lower than in other enamel layers near the dentine-enamel junction (Fig. 10). In the cusp layer especially, the highest density of crystals can be seen to be arranged regularly (Figs. 11, 12).

(a) Fine structures of the maturation stage of the ameloblasts (Fig. 13)

(1) Ameloblasts at the secretory stage

In the secretory stage of ameloblasts, the Golgi apparatus, round mitochondria and rough endoplasmic reticulum (ER) are situated proximally to the nucleus and in the distal column of the cytoplasm. Numerous multivesicular bodies and free ribosomes are observed distally. Secretory ameloblasts contain many bundles of microfilaments, running at right angles to the basement membrane to join the desmosome, at the lateral borders of the ameloblasts (Fig. 14). These lateral borders form the stellate reticulum. The infoldings of the cell interdigitaling surface, include many surface microvilli, particularly distally in the ameloblasts of this stage. The distal cell membrane of irregular thickness lies adjacent but not parallel to the unmineralized dentine surface. Tomes' process and terminal bars do not appear the cells (Fig. 15).

\section{(2) Maturation stage of the ameloblast}

In this stage, the elongate cells include numerous small round vesicles, small oval mitochondria and numerous oval rough ER (Fig. 16). The membrane at the lateral cell border closely apposes neighboring cells to join by underdeveloped desmosomes, and tight junctions appear in distally of the lateral border of the ameloblasts. The distal cell membrane, without a developed ruffled border, is closely associated with the enamel surface. Many filaments, parallel to the long axis of the tooth, appear in the distal portion of the cells near enamel-pedicel junction (Figs. 17, 18).

\section{(b) Distribution of the ATPase reaction in the secretory and maturation stages of the ameloblasts (Fig. 19)}

In the secretory stage of the ameloblasts ATPase activity is found chiefly in multivesicular bodies, round mitochondria and at the lateral borders of the cells, which are infolded and interdigitate. There is no reaction in the Golgi complex and rough ER (Figs. 19, 20). In the maturation stage, there is ATPase activity in numerous small round vesicles in the lateral borders of the ameloblast, forming the stellate reticulum, and at the infolded distal interdigitated surface membranes. Reaction product also occurs in multivesicular bodies at the distal cell membrane and enamel layer (Figs. 21, 22).

\section{Discussion}

The fine structure of the enamel layer of $\mathrm{Hyla}$ japonica is distinguishable as two layers, determined by the arrangement of the enamel matrices which show variations as seen under SEM and TEM. The outer layer in the cusp of the teeth resembles primitive enamel and the basal layer near the pedicel structurally resembles enameloid of the teeth of the salamander (Hynobius nigrescens) and the newt (Triturus pyrrhogaster). The enamel layer of Hyla japonica is comparable to that described in the cusp layer of the 
salamander and newts (Kawasaki and Fearnhead, 1983) in contrast to the basal layer near the pedicel. According to Fearnhead (1979), the enamel generally possesses three distinguishing features; (1) apatite crystailites, very much larger than those in mesodermally derived calcified tissues; (2) an organic matrix free from collagen; (3) when mineralization is complete, only minute residues of organic matrix remain in the tissue substance. The basal layer of Hyla japonica teeth near the pedicel, has very small crystals similar to the enamel crystals in the outer cusp layer; this feature resembles enameloid of other amphibians (Kawasaki and Fearnhead, 1983), whose enamel has no contain collagenous matrices in the basal layer near the pedicel. We conclude that the enamel of the basal layer near the pedicel is 'true enamel'. Enamel matrices of amphibians and some reptilians (Meredith Smith and Miles, 1971; Zaki and MacRae, 1977, 1978; Kawasaki and Fearnhead, 1983; Piesco and Kallenbach, 1985) do not form typical enamel rods. In the secretory stage of the ameloblasts of Hyla japonica cellular spaces develop between their lateral borders similar to those in larval and adult dental epithelial cells of the teeth of urodeles (Meredith Smith and Miles, 1971). In the young rat a terminal bar develops before formation of the dental matrices of the molar teeth. The distal basement membrane elongates near the terminal bar and forms the Tomes' process (Reith, 1970). In contrast, in Hyla japonica, as in other amphibians, the Tomes' process is not found. Tomes' processes thus are not essential for the pattern and arrangement of the enamel matrices in amphibians teeth in contrast to mammals, when enamel formation may well be related to the ruffled border and terminal bar of the ameloblasts. But in Hyla japonica, such components do not form in cells at the secretory stage. The distal portion with tight junction has an undeveloped lateral border before the formation of the enamel matrices. The enamel matrices, which do not differentiate "enamel rods", perhaps have no need for a Tomes' process present in mammals. However, the ameloblast of Hyla japonica has many multivesicular bodies in the secretory stage and small round vesicle in the maturation stage, as in other amphibian teeth (Zaki et al., 1970, Meredith Smith and Miles, 1971). In terms of the distribution of ATPase, these structures in the distal portion of the secretory cells may well be concerned with $\mathrm{Ca}$ transport. In the rat enamel organ, the cell membrane directs Ca towards the enamel layer; Ca permeates the intercellular space of the bands of smooth-ended ameloblasts and reaches the enamel by positive pressure (Crenshaw and Takano, 1982). In Hyla japonica during the secretory and maturation stages of the ameloblasts, ATPase activity is found in the lateral borders of the ameloblast forming the stellate reticulum and at the distal portion, and in the distal cell membrane and enamel layer. However, the density of reaction product ATPase in them is lower than that of mammals.

In amphibians, tight junctions at the distal region presumedly it isn't the entrance of $\mathrm{Ca}$ into the enamel matrices zone through the intercellular space of ameloblasts. Ca entry into this area is through the distal cell membrane actively by the sodium phosphate pump. In mammals, enamel rods form by $\mathrm{Ca}$ and matrix filaments, transported through the Tomes' process. Tomes' process contain many multivesicular bodies and small round vesicle where high ATPase activity occurs (Crenshaw and Takano, 1982). Nevertheless Ca transport, in Hyla japonica ameloblasts general, resembles that of mammals ultimately ATPase activity reaction, absent less extreme than in mammals, possibly related to the fact that the crystals are smaller and fewer in number in the maturation enamel layer of Hyla japonica. In Hyla japonica, also, transport of Ca occurs more gradually, the enamel layer is, in contrast, thinner than in mammals teeth.

\section{Acknowledgments}

We wish to thank Dr. Harold Fox (Department of Biology, Univ. College London) for reading the manuscript and advice on its research.

\section{References}

1) Ando, T., Fujimoto, K., Mayahara, H., Miyajima, H. and Ogawa, K.: A new one-step method for the histochemistry of Ca-ATPase activity. Acta histochem. cytochem., 14: 705-715, 1981.

2) Casey, J. and Lawson, R.: A histological and scanning electron microscope study of the teeth of caecilian amphibians. Archs. Oral Biol., 26: 49-58, 1981.

3) Crenshaw, M. A., Takano, Y.: Mechanisms by which the enamel organ controls calcium entry into developing enamel. J. Dent. Res., 61 (Sp Iss): 1574-1579, 1982.

4) Fearnhead, R. W.: Matrix-Mineral relationships in enamel tissues. J. Dent. Res., 58 (B) (Sp Iss): 909-916, 1979.

5) Ishida, T., Ogawa, Y. and Yagi, T.: Effects of fluoride on the enamel formation in rats. In Suga, S. (Ed), Mechanisms of tooth enamel formation, pp. 285-303. Quintessence Pub. Co., Inc., Tokyo, Berlin Chicago, Rio de Janeiro, 1983.

6) Kawasaki, K. and Fearnhead, R. W.: Comparative histology of tooth enamel and enameloid; in Suga S (Ed), Mechanisms of tooth enamel formation. Part III Mineralization, pp. 229-238. Quintessence Pub. Co., Inc, , Tokyo, Berlin, Chicago, Rio de Janeiro, 1983.

7) Meredith Smith, M. and Miles, A. E. W : The ultrastructure of odontogenesis in larval and adult urodeles: differentiation of the dental epithelial cells. Z. Zellforsch, 121: 470-498, 1971.

8) Piesco, N. P. and Kallenbach, E.: The fine structure of carly tooth formation in an lguanid lizard, Anoils carolinensis. I. Morph., 183: 165-176, 1985.

9) Reith, E. J.: The stages of amelogenesis as observed in molar teeth of young rats. J. Ultrastruct. Res., 30: 111-151, 1970.

10) Zaki, A. E. and MacRae, E. K.: Fine structure of the secretory and non-secretory ameloblasts in the forg. I. Fine structure of the secretory ameloblasts. Am. J. Anat., 148: 161-194, 1977.

11) Zaki, A. E. and MacRae, E. K.: Fine structure of the secretory 
and non-secretory ameloblasts in the frog. II. Fine structure of the non-secretory ameloblasts. J. Morph., 158: 181-198, 1978 .
12) Zaki, A. E., Yaeger, J. A. and Gillette, R.: Fine structure of the epithelial dental organ in the frog during early odontogenesis. Anat. Rec., 168: 79-92, 1970.

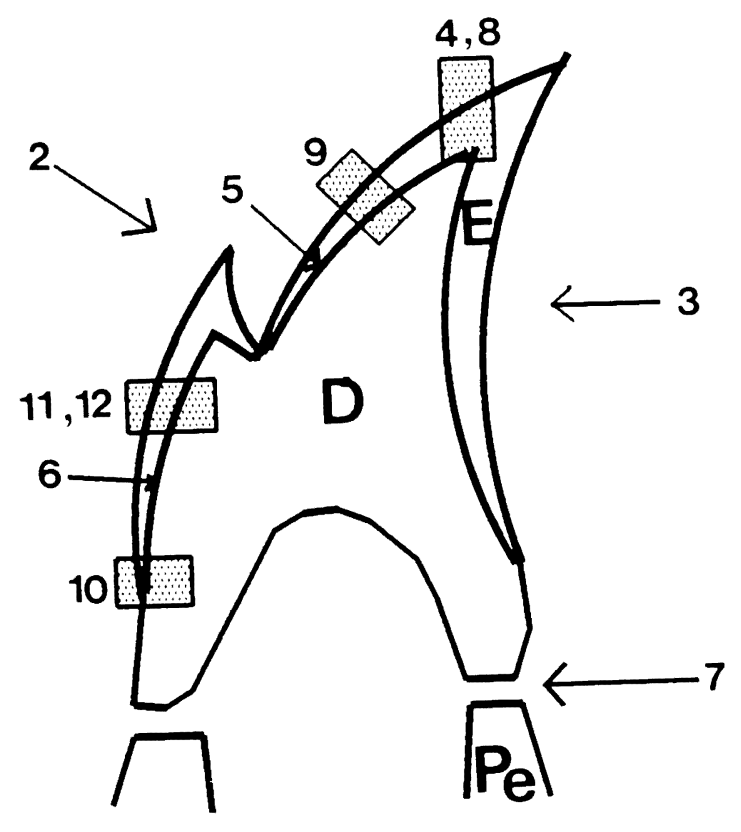

1

\section{Explanation of Figures}

\section{Plate I}

Fig. 1. Illustration of the teeth in a tree frog.

The observation is indicated by the square zone and arrows by Fig. 2 through Fig. 22 by SEM and TEM. Big arrow; direction of SEM observation, Square zone; SEM and TEM observations in this parts, D: dentine, E: enamel, Pe: pedicel.

\section{Plate II}

Fig. 2. SEM of the teeth of a tree frog.

The tooth of the lingual (ling.) and labial cusps (lab.), (Bar $=25 \mu \mathrm{m})$.

Fig. 3. The lingual surface of the lingual cusp appear rough of which small masses (arrow) occur (Bar $=5 \mu \mathrm{m}$ )

Fig. 4. The dentine (D)-enamel (E) junction in the apex of the lingual cusp ( $\mathrm{Bar}=1 \mu \mathrm{m}$ ).

Fig. 5. The dentine beneath the enamel layer near the lingual cusp of which oval masses (arrow) occur $(\mathrm{Bar}=1 \mu \mathrm{m})$

Fig. 6. The dentine layer near the pedicel. Many small oval masses (arrow) occur in this portion $(\mathrm{Bar}=1 \mu \mathrm{m})$.

Fig. 7. Bundles of collagen fibers (arrow) join the tooth (T) to the pedicel (Pe), (Bar $=10 \mu \mathrm{m})$. 
Structure and Histochemistry of Tree Frog Enamel 15

Plate II

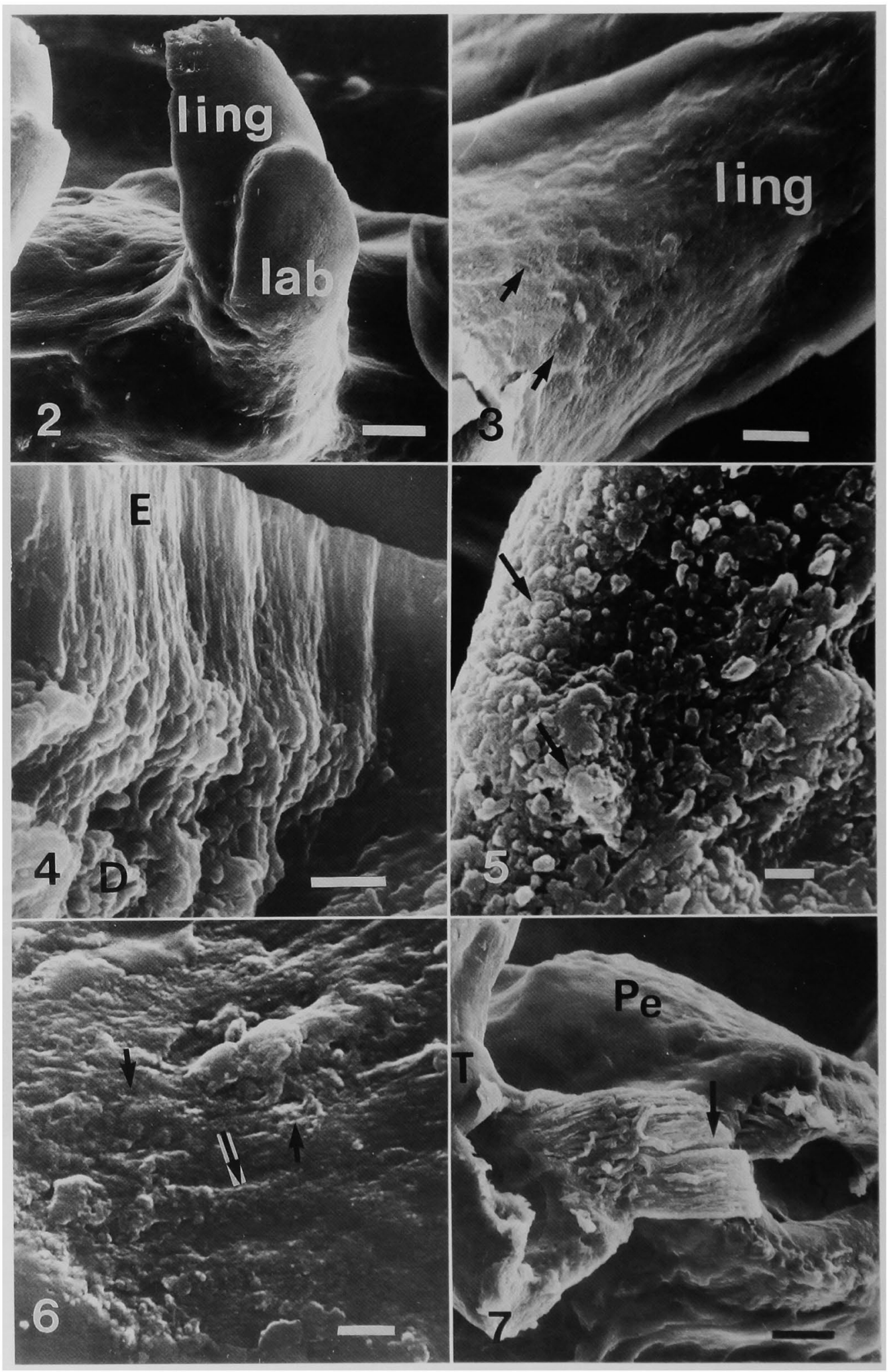


Plate III

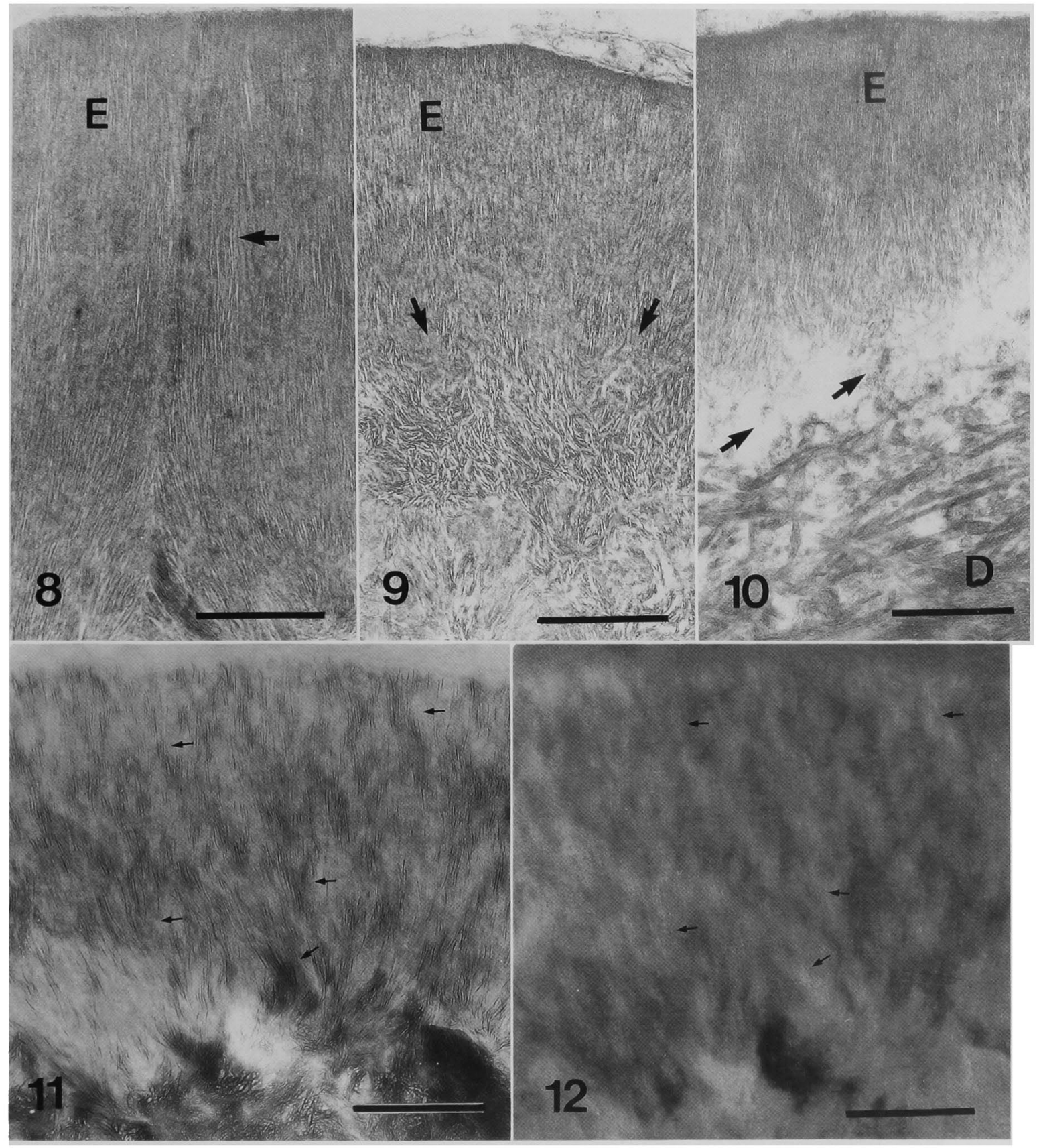

\section{Plate III}

Fig. 8. TEM of a longitudinal section of a tooth of the enamel layers $(\mathrm{Bar}=2 \mu \mathrm{m})$. In the enamel layer of the cusp, a mineral matrix contains crystals (arrow) oriented at right angles to the long axis.

Fig. 9. The basal layer near the pedicel; matrices (arrow) arrange irregularly (Bar $=2 \mu \mathrm{m}$ ).

Fig. 10. In the pedicel-enamel junction, dentine (D)-enamel (E) junction disappear (arrow) (Bar $=2 \mu \mathrm{m}$ ).

Fig. 11. The unstained enamel layer of the cusp with fine crystalline spindles (arrow) in this region $(\mathrm{Bar}=0.5 \mu \mathrm{m})$.

Fig. 12. A dark field image of Fig. $11(\mathrm{Bar}=2 \mu \mathrm{m})$. 

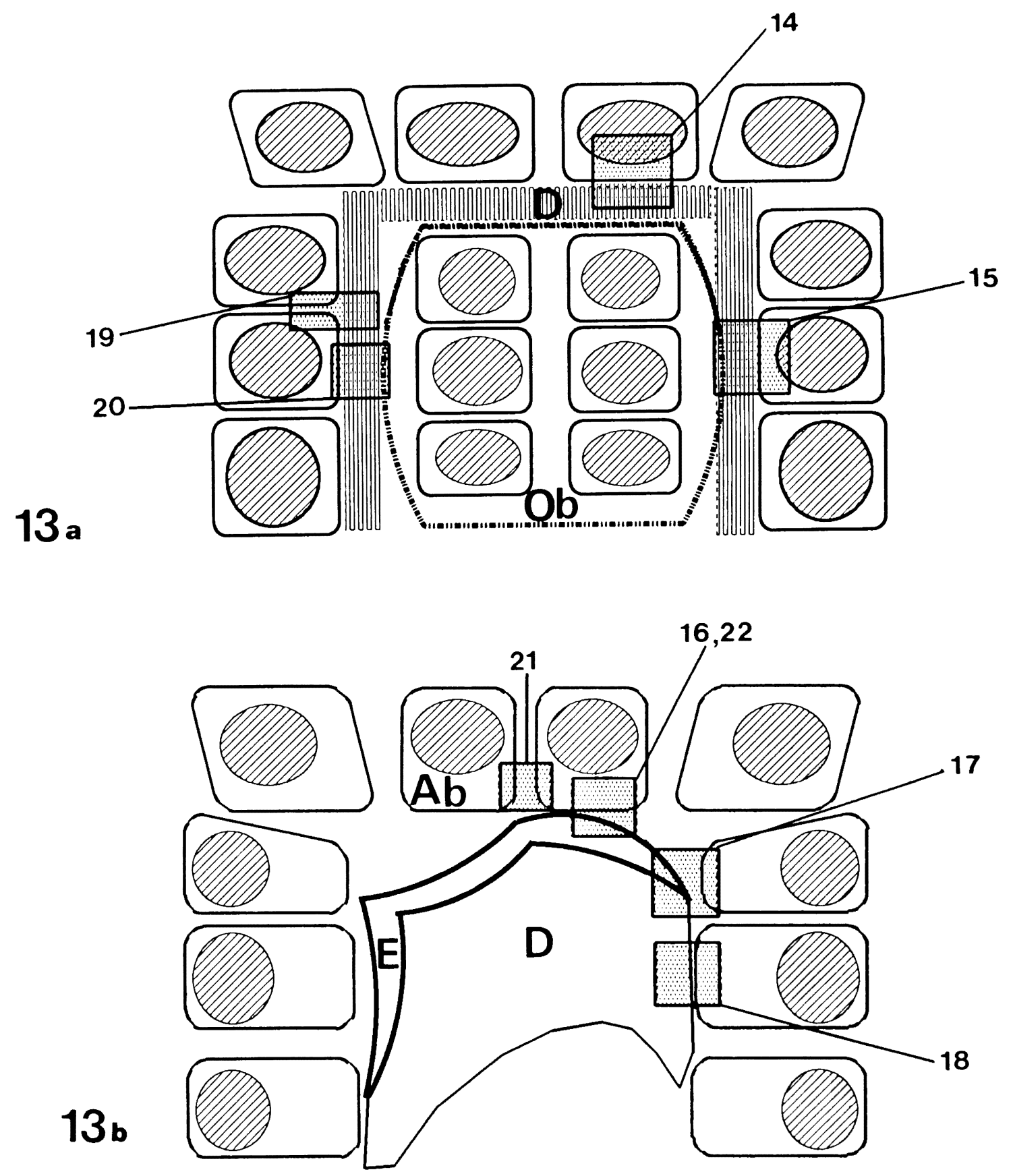

Plate IV

Fig. 13. Illustration of the teeth germ in a tree frog.

a: The observation is indicated by the square lines by Fig. 14 through 18 by TEM in the secretory stage of the ameloblasts.

b. The observation is indicated by the square lines by Fig. 19 through 22 by TEM in the maturation stage of the ameloblast. Ab: ameloblast, Ob: odontoblasts, D: dentine; E: enamel 
Plate V

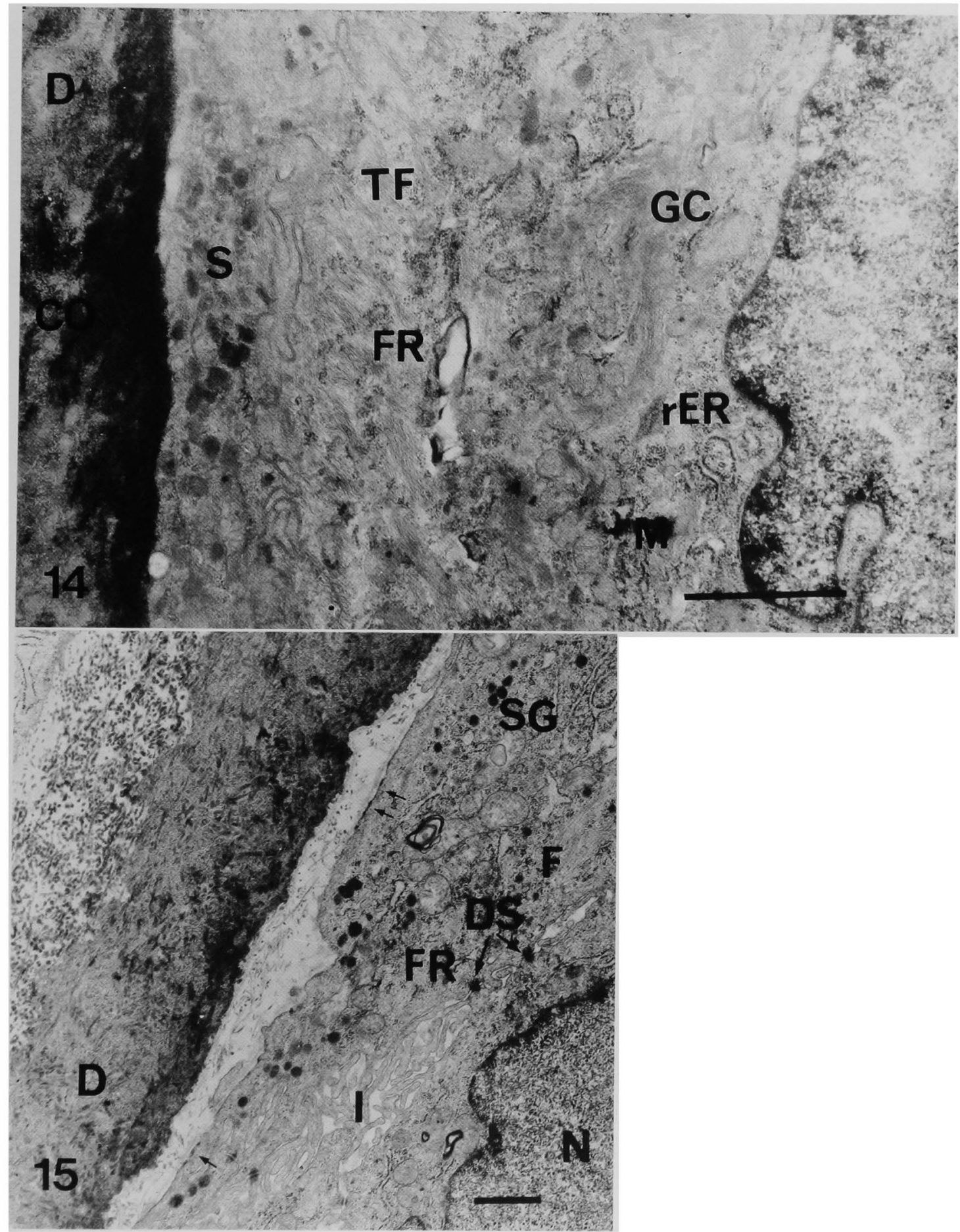

Plate V

Fig. 14. TEM of a longitudinal section of a ameloblast in the secretory stage.

The secretory stage of a cell contains many small granules (SG), tonofilaments (TF), free ribosome (FR), oval mitochondria (M), Golgi complex and tubular rough surface endoplasmic reticulum (rER) near the nucles $(\mathrm{N})$. Collagenous matrices $(\mathrm{CO})$ increase in the dentine layer (D) $(\mathrm{Bar}=1 \mu \mathrm{m})$.

Fig. 15. The developed interdigitation (I), desmosome (DS) is found in the distal portion of cell. The apical cell border of ameloblast shows area of increased density (arrow) $(\mathrm{Bar}=1 \mu \mathrm{m})$. 
Plate VI
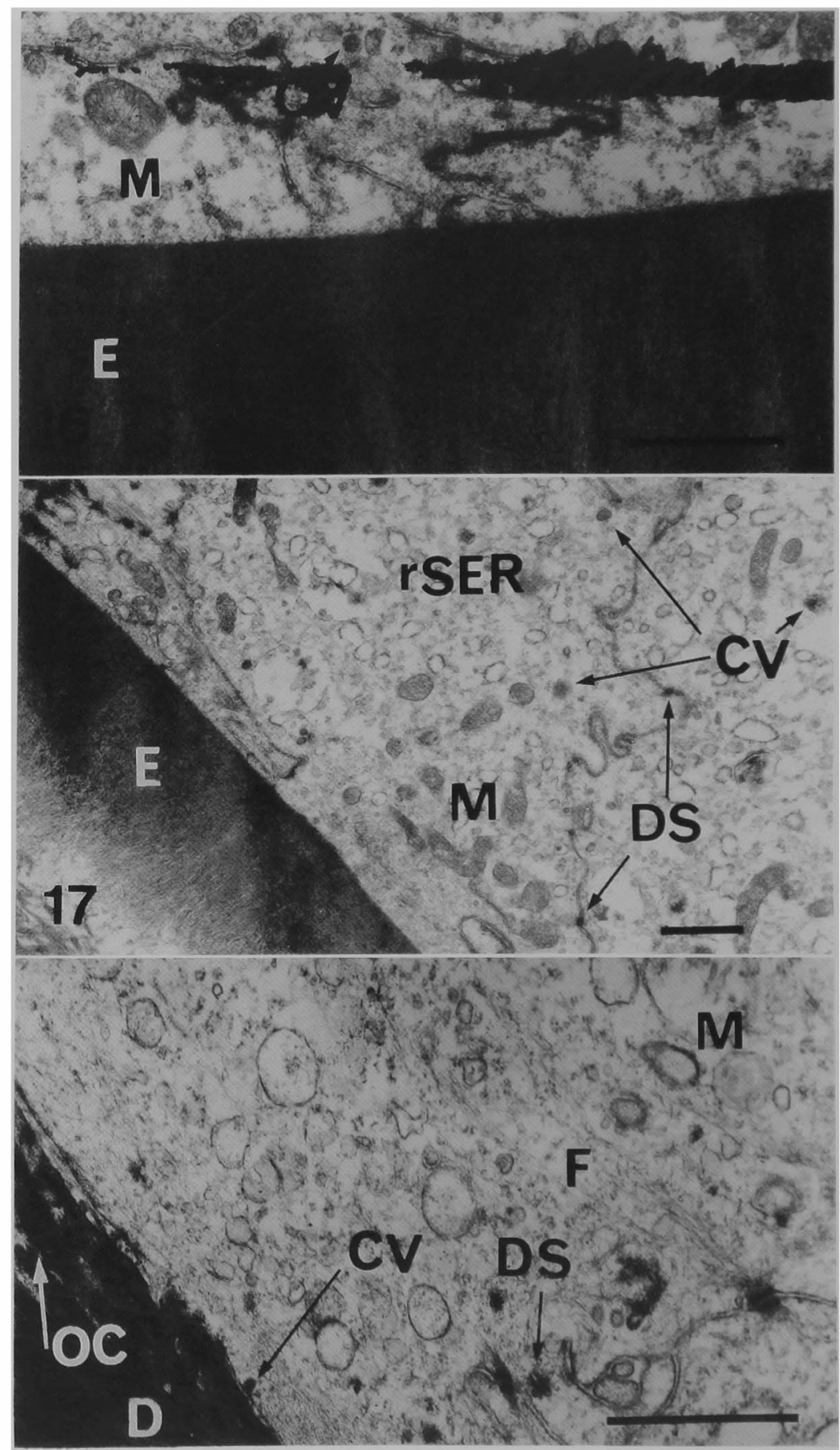

\section{Plate VI}

Fig. 16. TEM of a longitudinal section of a ameloblast in the maturation stage. The ameloblast contains small oval mitochondria and coated vesicle near the enamel layer $(\mathrm{E})$ in the maturation stage $(\mathrm{Bar}=1 \mu \mathrm{m})$.

Fig. 17. Many oval rough surface endoplasmic reticulum (rSER) are found in the cell at the distal portion $(\mathrm{Bar}=1 \mu \mathrm{m})$.

Fig. 18. In the basal portion of the teeth, many small fibers $(F)$ and vacuole are found near the dentine layer (D) having fibrous matrices $(\mathrm{OC})(\mathrm{Bar}=1 \mu \mathrm{m})$ 
Plate VII

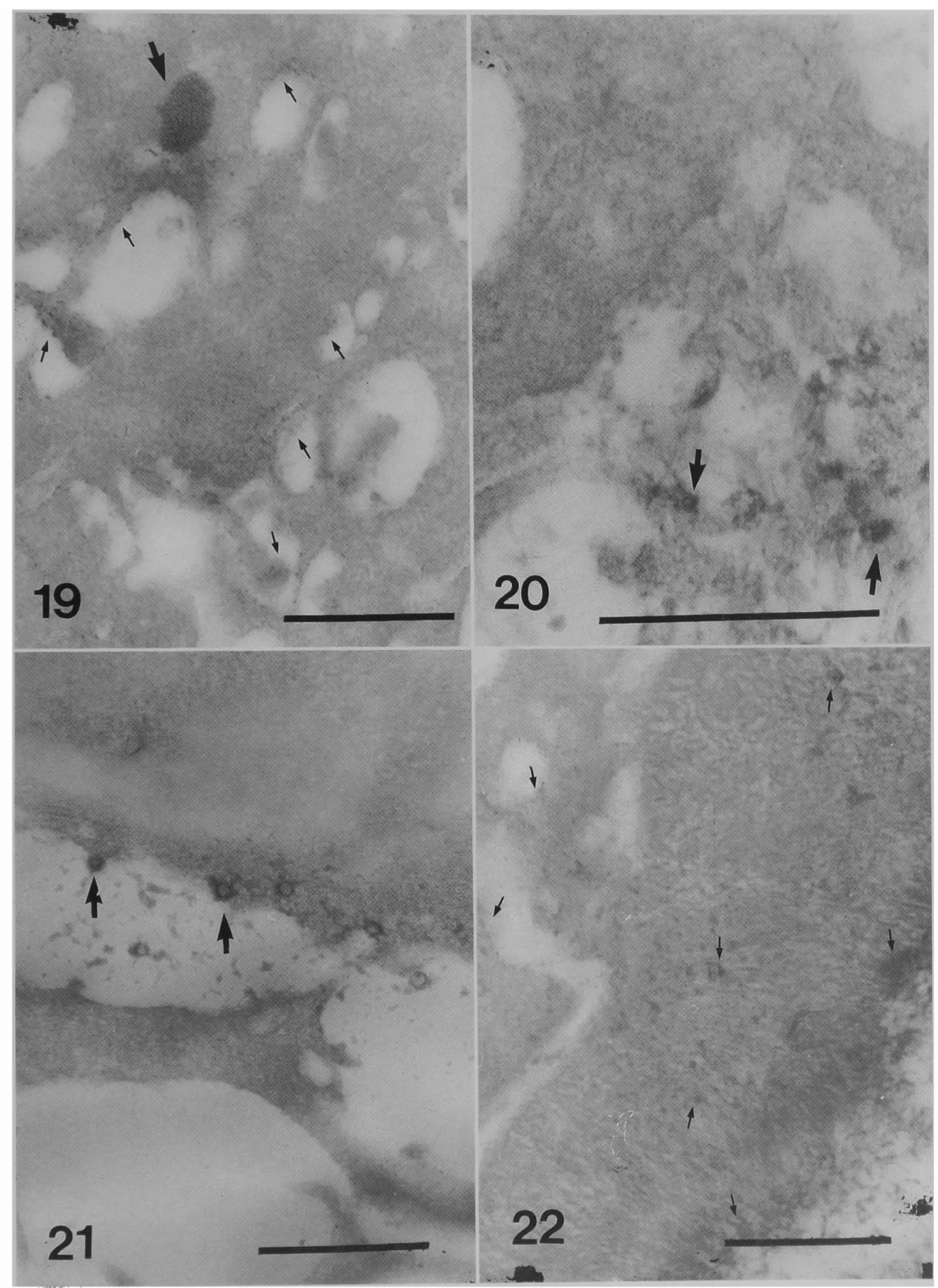

\section{Plate VII}

Fig. 19. TEM of a longitudinal section of a ameloblast in a ATPase activity. At the distal portion of the cell in the secretory stage, the reaction occur in the intercellular space, secretory granules, mitochondria (arrow) $(\mathrm{Bar}=1 \mu \mathrm{m})$.

Fig. 20. At the basement membrane of the cell in the secretory stage, the reaction occur in the small coated vesicle (arrow) $($ Bar $=1 \mu \mathrm{m})$.

Fig. 21. At the distal portion of the cell in the maturation stage, the reaction is more strong in the intercellular space, small oval multivesicular body (arrow) $(\mathrm{Bar}=1 \mu \mathrm{m})$.

Fig. 22. In the dentine-enamel junction, reaction occur in the distal cell membrane and enamel matrices (arrow) $(B a r=1 \mu \mathrm{m})$. 